\title{
PREJUICIOS IMPLÍCITOS Y EXPLÍCITOS DESDE ESTUDIANTES UNIVERSITARIOS HACIA ADULTOS MAYORES
}

Cristóbal Ramírez Monsalve - Universidad Católica Silva Henríquez

Cristóbal Ramírez Monsalve

\section{Correspondencia: \\ Cristóbal Ramírez \\ General Jofré 462. Santiago, Chile. \\ Fono 224601235, +56 978058738 \\ cramirezm@miucsh.cl}

Agradecimientos:

Quisiera agradecer al profesor que aporto en esta publicación, el académico Carlos Ascencio junto a los compañeros(as) Errol Aguilera, Constanza Sepúlveda y Carol Soto por participar previamente en la investigación durante el seminario de grado, y finalmente a la Universidad Católica Silva Henríquez por su aporte frente a nuestra formación educacional. 


\title{
Resumen
}

El presente artículo presenta un estudio de prejuicios en sus dimensiones implícitas y explicitas de estudiantes universitarios hacia adultos mayores. El levantamiento de información se realizó por medio de entrevistas semi-estructuradas, un grupo focal y el Test de la figura humana. El análisis de datos se desarrolló a través de una codificación abierta y axial. Los resultados arrojaron que los prejuicios disminuían cuando existía un grado de cercanía a familiares o conocidos de la tercera edad; además hubo una variación según el tipo de carrera, pues las carreras asociadas a salud apelaron a una opinión de los adultos mayores que difería de las carreras del ámbito social comunitario; también existió una variabilidad en la elaboración de las respuestas relacionado al rango de edad de los participantes.

Palabras clave: prejuicio; universitarios; adultos mayores; representaciones sociales

\begin{abstract}
This article presents a study of prejudices in their implicit and explicit dimensions of university students towards elderly people. Information gathering was carried out through semi-structured interviews, a focus group and the Human Figure Test. Data analysis was developed through open and axial coding. The results showed that prejudices decreased when there was a degree of closeness to family members or acquaintances of the third age, in addition there was a variation according to the type of career, since the careers associated with health appealed to an opinion of older adults that differed from community social careers; There was also a variability in the elaboration of the answers related to the age range of the participants.
\end{abstract}

Key words: prejudice; university students; elderly people; social representations 


\section{Introducción}

La vejez es una etapa del ciclo vital que demográficamente aumenta rápidamente, ya que como menciona Thumala (2011, p. 2) “en los próximos treinta años aproximadamente una de cada cuatro personas será adulto mayor". Pensando esto en un contexto neoliberal donde el objetivo es producir, se desarrolla un pensamiento individualista y competitivo que además rige la mentalidad del sujeto actual, lo cual se visualiza como una problemática. Los individuos con características más afines al sistema tienen ciertas características, "una de estas refiere a la posición de superioridad en la que se sitúan estos otros grupos que no pertenecen a la vejez, viéndose a sí mismos como máxima autoridad” (Araya \& Villena, 2006, p. 27)

Es a partir de esta actitud que se emiten juicios morales, sociales, políticos, culturales y legales que terminan por dañar a otras personas. Estos grupos no pertenecientes al estereotipo de adulto mayor, se caracteriza por la atribución de aspectos positivos hacia sí mismos, por lo que suelen considerarse más capaces, mejores, con mayor aptitud intelectual e incluso pertenecientes a estructuras sociales y organizaciones políticas superiores.

Dentro de la sociedad se encuentra una diversidad de sujetos marginados socialmente, pero sobre todo el grupo de adultos mayores. Estos individuos son prejuiciados y discriminados no solo explícitamente, sino además implícitamente desde las actitudes de otras personas guiadas por una representación social hacia ellos. Sufren conductas discriminatorias en la interacción cotidiana por otros grupos, los cuales perciben a las personas mayores como incapacitadas físicamente, sufrir pérdidas ambientales y ser económicamente dependientes. Ello a su vez “constituye una barrera para la participación en el mercado de trabajo y es una forma de exclusión social relacionada con el derecho a un ingreso y la participación económica en diferentes instituciones y ámbitos sociales" (Osorio, 2008, p. 224). 
En este marco se presenta el trabajo, el cual expone principalmente los prejuicios implícitos y explícitos teniendo a la base las representaciones sociales hacia los adultos mayores. Las cuales se presentan de manera preponderante con aspectos negativos, lo que a su vez los desfavorece, y en consecuencia reduce su participación activa dentro de la sociedad, segregándolos y provocando la aparición de prejuicios y discriminación. Las consecuencias e implicancias en la interacción con el sistema social, da cuenta que adoptan un rol negativo estereotipado, lo que causa la pérdida prematura de su independencia, una mayor discapacidad, mayores índices de depresión y una mortalidad anticipada. (Losada Baltar, 2004).

Las representaciones sociales que giran en torno a la vejez son predominantemente negativas y cargadas con un componente de discriminación y desigualdad. Evidenciando en esta afirmación la perjudicial carga negativa, se depositan en los adultos mayores características como: pasividad y dependencia, representándolos como sujetos obsoletos e inactivos (Jorquera, 2010).

Dentro de esta dinámica es que emerge el prejuicio, el cual es una conducta anticipada y que con el tiempo pasará a ser parte de una discriminación, y es considerado como una antipatía basada en una generalización inflexible y errónea, la cual puede ser expresada de manera explícita o sentida implícitamente (Ungaretti, Etchezahar \& Simkin, 2012). Siguiendo esta lógica es posible decir que el prejuicio se mantiene a la base de un pensamiento y actitud que se construye y está presente en la cultura. Además, se sostiene la idea de que el prejuicio sería un mecanismo intra-psíquico que los individuos crearían para recalcar las diferencias inter-grupales, lo que provocaría reacciones negativas hacia aquellos que no pertenecen al grupo en cuestión (Ungaretti, Etchezahar \& Simkin, 2012). 
Con lo dicho anteriormente se puede decir que el prejuicio al ser un mecanismo formado a nivel intra-psíquico, se ve reflejado en actitudes que delatan el contenido que funciona de manera inconsciente, las cuales para efectos de este estudio fueron denominadas implícitas, ya que "las actitudes no necesitan ser accesibles conscientemente para producir evaluaciones sobre un objeto; más aún, a menudo estas evaluaciones se realizan de modo inconsciente." (MontesBerges, 2006, p. 4)

Las actitudes provenientes del prejuicio constituyen una forma de discriminación social, dentro de estas se encuentra la intención de contacto reducido, lo cual se entenderá como una forma de manifestación conductual implícita y que actúa como una modalidad de expresión ocultativa de sentimientos negativos a los individuos de los exogrupos, no manifestándose abiertamente, sino más bien con una evitación de contacto. "La intención de contacto con el exogrupo se ha concebido, teórica y operacionalmente, como un constructo unidimensional definido básicamente por la intimidad" (Angosto \& Martínez, 2004, p. 182)

Como se ha mencionado, los sesgos negativos sobre la vejez son uno de los fenómenos característicos que enfrentan los adultos mayores, y a esto se le añade la presencia del edadismo, el cual hace parte de una orientación perjudicial. Este es entendido por Palmore (1990, en Losada Baltar, 2004) como una consecuencia de los estereotipos, el prejuicio y la discriminación, este da paso a actitudes prejuiciosas hacia las personas mayores sólo por el hecho de ser mayores. Además de ello, se conforman visiones altamente exageradas en donde las características positivas del individuo quedan omitidas.

Así mismo fomenta la discriminación, provocando la llamada muerte social, un fenómeno que prejuicia y segrega a los adultos mayores y afecta directamente su calidad de vida. En palabras de Osorio (2008, p. 224): "la discriminación por edad constituye una barrera para la 
participación en el mercado de trabajo y es una forma de exclusión social relacionada con el derecho a un ingreso y la participación económica en diferentes instituciones y ámbitos sociales".

Ahora, estas creencias a la base de conductas podrían no ser manifestadas por los propios sujetos, ya que existen situaciones de demandas sociales que impiden generar un daño al otro, ello se conceptualiza como deseabilidad social, lo cual es un "sesgo en la tendencia de respuesta cuyo objetivo es dar una imagen positiva de uno mismo" (Domínguez, Aguilera, Acosta, Navarro \& Ruiz, 2012, p. 809). Lo anteriormente descrito da paso a la conformación de prejuicios implícitos, los cuales afectan el vínculo y la comunicación con los propios adultos mayores, debido a la creación anticipada de una imagen equivocada hacia ellos. Estas tienen su origen en el contexto donde se inserta el sujeto, ya que las personas interpretan la realidad de acuerdo a ideas que emergen de forma espontánea al percibir estímulos. Los prejuicios implícitos se manifiestan a través de respuestas automáticas, las cuales son producto de un sistema cognitivo donde parecen ser codificadas dependiendo de los estereotipos o características (Montes- Berges \& Moya, 2006).

Otra forma de manifestación de conductas implícitas es el manejo de la impresión, aquí el cambio en las proyecciones de la autoimagen es el principal protagonista, ocasionando en las personas cambios intencionales en su comportamiento y actitud para lograr un fin determinado, en este caso, los participantes intentaron generar un manejo de la impresión opinando de manera favorable hacia el adulto mayor, lo cual fue decayendo con el curso de la entrevista, para revelar prejuicios que muchas veces contradecían el discurso inicial. Por lo tanto, el sujeto manipula de manera estratégica la información transmitida al otro (Acosta \& Domínguez, 2014). Lo cual 
influye en las actitudes que se transmiten hacia los adultos mayores, siendo estos prejuiciados con mecanismos ocultativos y paralelamente segregándolos de la esfera social.

Esta discriminación proveniente del prejuicio y de las construcciones sociales negativas hacia la vejez, caen en la ambivalencia de juzgar a otro sin conocerlo ni demostrar interés en ello, pero a su vez, expresándolo de igual manera. Esta poca coherencia es fruto de la dinámica de prejuiciar a los exogrupos, en donde la persona disfraza esto para actuar de otras maneras más sutiles, siendo esta una de las modalidades que más afecta al ámbito psicológico de los adultos mayores (Zavala, Vidal, Castro, Quiroga \& Klassen, 2006). Es por esta razón que los prejuicios hacia la vejez pueden evidenciarse de maneras tanto implícitas como explícitas, consiguiendo afectar el vivenciar y la autopercepción de los propios adultos mayores, como también la percepción y conducta que otros pueden adoptar hacia ellos.

Visibilizando los prejuicios que afectan a los adultos mayores, y exponiendo como una parte de la sociedad los segrega sutilmente, se pretende generar a partir de su reconocimiento un cambio de perspectiva hacia las personas mayores, no subestimando su aporte, sino más bien incluyéndolos, ya que también son parte importante de la sociedad.

Esta investigación revelo que existe una falta de coherencia en el discurso de los universitarios en relación a la vejez, esta falta de congruencia conduce inevitablemente a emitir prejuicios sin ser consciente de ello, puesto que esta discriminación está internalizada y emerge a modo de prejuicios implícitos en el discurso, mostrando facetas de los adultos mayores cargadas de aspectos negativas.

Cabe destacar que, pese a la predominancia de prejuicios negativos en la población de universitarios jóvenes, también existieron visiones positivas hacia la vejez, las cuales se 
originaron en los participantes que convivieron alguna vez con un hombre o mujer mayor a 65 años de edad en su hogar (Thumala, Arnold, Massad \& Herrera, 2015).

Finalmente, al exponer los resultados de la investigación en el presente artículo, se busca generar una discusión reflexiva de manera crítica en relación a las representaciones sociales a cerca de la vejez, con la finalidad de derribar aquellos prejuicios que mantienen la discriminación y en consecuencia la exclusión hacia este grupo minoritario; concientizando a las personas y específicamente al lector en torno a las situaciones que atraviesan las personas mayores y los efectos negativos que generan en su vida.

\section{Método}

Se optó por el método cualitativo, implementando además la perspectiva epistemológica de tipo fenomenológica la cual explica los fenómenos entregados por la conciencia, así mismo "la fenomenología es un intento sistemático de develar las estructuras significativas internas del mundo de la vida” (Rodríguez, Gil \& García, 1996, p.40)

Al utilizar la perspectiva fenomenológica se entendió la realidad social desde un enfoque constructivista, es decir, como algo que está continuamente en construcción. De este modo, los sujetos son agentes partícipes de ello, son ellos mismos los principales actores sociales y los cuales modifican y dan significado al mundo en el cual habitan.

También se desarrolló un muestreo teórico, este tipo de proceso investigativo como afirma Glasser \& Strauss (Citado en Flick, 2007) "Es un proceso de recogida de datos para generar teoría por medio del cual el analista recoge, codifica, y analiza sus datos conjuntamente"

En cuanto al lugar y los participantes, se escogió la Universidad Católica Silva Henríquez debido a la visión comunitaria integrada en la formación de profesionales, sensibilizados en el área de vulnerabilidad y exclusión. Además esta institución presenta una población estudiantil 
mayoritariamente asociada al nivel socio económico C3, es decir, a una de las categorías que clasifican el nivel socioeconómico de cada familia, el cual y en base al estudio realizado por el Servicio Nacional del Adulto Mayor (2013) titulado Inclusión y Exclusión Social de las Personas Mayores en Chile, menciona que dicho nivel presenta mayores conductas discriminatorias en comparación a otras categorías, así mismo indica que el hecho de convivir o no con adultos mayores afecta en la cantidad de discriminación encontrada. En cuanto a la elección de las carreras, ésta fue desarrollada en torno al fundamento de una futura relación directa con pacientes o clientes pertenecientes a la vejez.

De acuerdo a los parámetros de selección, los participantes presentaron las siguientes características:

1. Estudiantes de la Universidad Católica Silva Henríquez de nacionalidad chilena, pertenecientes a las carreras de kinesiología, enfermería, fonoaudiología, psicología, derecho, trabajo social, sociología y pedagogía en educación física.

2. Pertenecientes a las comunas de Maipú, Ñuñoa, Macul, Peñalolén, La Florida, San Joaquín, Estación Central, Recoleta, Independencia, Puente Alto y San Bernardo.

3. Que no convivan con adultos mayores en su domicilio.

En el presente estudio se desarrolló una estrategia de levantamiento de información que consistió en una triangulación de métodos. Se utilizó el test de la figura humana, el cual para fines de esta investigación se modificó solicitando al entrevistado que dibujara primero un “adulto mayor" sin mayor sugerencia o indicación que pudiera perturbar el proceso. Lo anterior permitió la asociación para la aclaración de significados individuales (Portuondo, 2007). Cabe destacar que se utilizó la figura humana y su adaptación de manera paralela, con la finalidad de 
indagar en profundidad los aspectos implícitos de los participantes, en relación a la vejez, analizando y comparando el dibujo de la figura humana y la del adulto mayor esbozado por estos.

Luego, se implementó una entrevista semi estructurada en la cual el entrevistador tuvo la libertad de introducir preguntas adicionales para precisar conceptos u obtener mayor información sobre los temas deseados; es decir, no todas las preguntas estaban predeterminadas (Hernández, Fernández \& Baptista, 2006). Este método tuvo una duración de aproximada de 30 a 50 minutos y estuvo compuesta de tres partes: 1) Se realizaron catorce preguntas en las cuales se debían completar frases; 2) Casos hipotéticos; 3) Una postura personal hacia la vejez.

Durante el desarrollo, se realizó la siguiente consigna: “A continuación, te vamos a hacer una serie de preguntas rápidas, donde tienes que escoger entre dos opciones, en el menor tiempo posible y luego debes argumentar el porqué de tus respuestas".

En la tercera parte, se transmitió al entrevistado lo siguiente: “Ahora vamos a presentarte distintas situaciones, para saber cómo responderías si hipotéticamente te pasaran a ti”.

Finalmente se realizó un grupo focal el cual tuvo una duración de una hora aproximadamente y reunió a siete personas en un día y lugar determinado, lo que fue acordado previamente con los estudiantes que participaron y quienes a su vez contaban con la particularidad de no haber participado en la entrevista individual. En el desarrollo del grupo focal se realizaron 12 preguntas relacionadas a la vejez, con la finalidad de recabar las diferentes representaciones sociales hacia esta etapa, y donde además se procuró incidir en el diálogo y el debate entre los participantes para generar una mayor extracción de datos; todo este proceso fue registrado en un audio. 
El levantamiento de información finalizó con la triangulación de métodos distintos, deteniéndose así cuando existiese una saturación teórica; es decir, cuando los datos se repitieran sistemáticamente. La estrategia de análisis se llevó a cabo por medio de la teoría fundamentada, la cual "utiliza una serie de procedimientos que, a través de la inducción, genera una teoría explicativa de un determinado fenómeno estudiado, en este sentido, los conceptos y relaciones entre los datos son producidos y examinados continuamente hasta la finalización del estudio" (Cuñat, 2007, p. 1). El análisis, parafraseando a Flick (2007), se abordó con la codificación abierta, la cual ayudó a expresar los datos y los fenómenos en forma de conceptos, para luego efectuar la codificación axial con la que se filtraron y diferenciaron las categorías derivadas de la categorización expuesta anteriormente. Con ello, se efectuó un análisis descriptivo e interpretativo, los cuales contribuyeron a esclarecer las representaciones sociales hacia la vejez y los factores que intervienen en la aparición de estos, junto con los niveles implícitos y explícitos del discurso.

Cabe destacar que, en la Entrevista individual y en el grupo focal, se facilitó un consentimiento informado diseñado sobre la base del código de ética del Colegio de Psicólogos de Chile, con el fin de resguardar la confidencialidad de los participantes, ya que "La documentación y difusión de la actividad profesional del psicólogo/a no debe transgredir los principios y normas de confidencialidad a que está obligado" (Código de Ética Profesional, 1999, p. 10).

\section{Resultados}

La información obtenida sugirió que existen representaciones sociales positivas y negativas de la vejez, esto producto de la discriminación inserta en el contexto actual a causa de prejuicios por la falta de desmitificación sobre temáticas asociadas a los adultos mayores. Los factores detectados en las respuestas de los entrevistados y participantes del grupo focal son parte de la 
interpretación y reflexión de los investigadores, estas a su vez se desglosaron en: 1) edad; 2) tipo de carrera; 3) la experiencia previa con adultos mayores.

En este sentido, la edad sería un factor clave a la hora de evaluar la riqueza y elaboración de respuestas frente a preguntas que podrían sugerir cierta abstracción y profundidad, al enfrentarse a situaciones en que estaría en juego la deseabilidad social frente a un extraño.

Por otro lado, se pudo apreciar por parte de los investigadores que las carreras relacionadas al área de salud presentan una mayor presencia de prejuicios negativos hacia la vejez implícita y explícitamente, debido a la formación y mallas curriculares donde abarcan temáticas de índole más biológica. Por otra parte, la carrera de Derecho basó su juicio en el modo de ejercer la docencia de sus profesores adultos mayores, percibidos como un sinónimo de poder autoritario e inflexibilidad.

Para el caso de carreras asociadas a las ciencias sociales, existió una mayor variedad de respuestas, sin embargo, de igual manera se visibilizaron prejuicios, sólo que en un carácter más implícito. De igual manera, en esta área se consideró el hecho de que un pequeño grupo de los participantes tuvo una experiencia en los últimos años con adultos mayores e incluso vivencias negativas frente a familiares pertenecientes a este grupo etario, esta nueva variable en comparación a otras áreas, contribuyo a una mayor heterogeneidad en las respuestas.

Asimismo, de acuerdo al análisis interpretativo, la información obtenida sugiere la existencia de indicadores tales como: ambivalencias, silencios, contradicciones y gestos físicos que en este artículo serán parte del llamado Manejo de la Impresión, entendida como "un conjunto de procesos psicológicos que le permiten al individuo controlar efectivamente las imágenes personales que proyecta al exterior mediante la selección de información y su control 
conductual y emocional permitiéndole alcanzar objetivos personales" (Acosta \& Domínguez, 2014, p.1).

Los indicadores obedecieron a un "manejo de la impresión" a causa de la deseabilidad social y son resultado de los mecanismos de defensa presentes en cada sujeto. Además, se observaron prejuicios positivos y negativos frente a la vejez, en donde continuamente existieron indicadores de prejuicios implícitos de carácter negativo, mientras que en pocas ocasiones estos eran concientizados por los entrevistados, aludiendo a una dimensión implícita inconsciente que enmascara el prejuicio para no ser revelado de manera explícita.

En cuanto a prejuicios positivos y negativos, implícitos y explícitos hacia los adultos mayores, la gran mayoría de los participantes hizo alusión a características psicobiológicas. En el caso de los prejuicios positivos, se representó como una etapa natural, que todos alcanzaremos en algún momento y se destacaba la gran sabiduría de quienes la cruzan, la capacidad de seguir ejerciendo labores remuneradas junto a la cercanía familiar. En cuanto a ganancias personales, está el presenciar la descendencia familiar, conocer nuevas redes de apoyo y principalmente de índole cognitiva que brindaría una ventaja frente al resto de grupos etarios al momento de analizar situaciones y problemáticas que alguien joven no podría resolver, como, por ejemplo, frente a temáticas de crianza orientadas hacia padres primerizos siendo una figura de apoyo con "mucho que entregar" y ser "un libro abierto" a la hora de educar a otros. Esto sería percibido como una suerte de "estatus" o "privilegio" frente a otros y representaría las características más deseables a la hora de pensar en la propia vejez. La mayoría de los prejuicios positivos se observaron de manera explícita en el discurso de los participantes.

Por otro lado, en cuanto a prejuicios negativos sobre la vejez, emergieron las representaciones sociales de enfermedad, abandono y estar incapacitados para ejercer labores 
remuneradas como no remuneradas. Es interesante destacar este punto, debido a la contradicción existente con la postura mencionada anteriormente, donde se destaca que serían personas capacitadas para ejercer labores relacionadas a un desempeño profesional.

Si bien el cuerpo se deteriora biológicamente, esto no sería un sinónimo de enfermedad como la mayoría de participantes argumentó en la entrevista personal y focus group. Pocas personas hicieron referencia al desgaste biológico como la presencia de arrugas y canas, más bien en su discurso predominó la relación de enfermedad y cercanía a la muerte.

Esta misma representación social de los adultos mayores como enfermos se conectó con una categoría que emergió a menudo en la entrevista semiestructurada, el hecho de categorizarlos como "un cacho", que, en otras palabras, significa ser una molestia para la familia o cuidador/es, por lo que finalmente acaba en abandono y por ende en muerte social. La mayoría de los prejuicios negativos se observaron de manera implícita en los participantes, con la excepcionalidad de dos participantes, los cuales concientizaron al final de la entrevista la cantidad de prejuicios que tenían acerca de la vejez de forma explícita.

Se evidenció que los prejuicios negativos implícitos y prejuicios positivos explícitos se expresaban con una dinámica general, es decir, el discurso con prejuicios positivos aparecía al principio de la entrevista, y luego durante el transcurso de la entrevista y a medida que las defensas del participante bajaban se daba un fenómeno en el que el participante contradecía su postura inicial respecto a la imagen de adulto mayor que tenía de acuerdo a su representación social personal de la vejez.

Finalmente, se puede concluir que se hace interesante la coexistencia de dos aspectos contradictorios y/o ambivalentes dentro del discurso de una misma persona, los cuales se develan en el transcurso de la entrevista al verse enfrentados a distintas situaciones desde lo más lejano, 
como por ejemplo, su opinión personal hacia la vejez y finalmente enfrentarlo ante el propio e inevitable envejecimiento del ser humano, siendo este el punto alto de aparición de los prejuicios negativos implícitos hacia la vejez.

Las contradicciones en la entrevista semi-estructurada serían entonces un indicador característico en este caso de la existencia de prejuicios implícitos hacia la vejez, así como los silencios y ambivalencias. El hecho de mostrarse indeciso frente a preguntas socialmente comprometedoras, sería a nivel interno un despliegue de mecanismos defensivos los cuales coartan el discurso en los jóvenes estudiantes. Esto es propiciado por la necesidad de adecuarse a la situación, ya que frente a la duda de ser juzgado es preferible recurrir a un silencio mientras el sistema cognitivo trabaja por elaborar una respuesta considerada "adecuada" que más tarde, a medida que transcurre la entrevista, se desvanece y permea opiniones totalmente contrarias a las iniciales.

Entre las diferencias principales de los Dibujos de la Figura Humana de Machover (figura humana general) y el de innovación metodológica del adulto mayor, se observó que en su mayoría la figura humana general presentaba alguna parte de su cuerpo en movimiento, predisposición a la acción o practicando algún tipo de deporte, a diferencia de los adultos mayores quienes son representados inmóviles, rígidos y con dificultad en la movilidad, ayudándose de un bastón, lo que daría cuenta de que se deposita en ellos algún tipo de incapacidad o pasividad como principales características. También se encontró evidencia de una representación social de infantilismo hacia los adultos mayores, demostrado en su mayoría con botones en su vestimenta. En contraste con los dibujos de la figura humana general, los cuales carecen de accesorios en la ropa. 
A través del grupo focal, fue posible observar con mayor frecuencia el levantamiento de defensas frente a la conservación de una imagen socialmente aceptada en función de la deseabilidad social y el enmascarar los prejuicios desfavorables hacia los adultos mayores. A la hora de emitir opiniones frente a preguntas sobre la propia vejez es donde más se presenta este fenómeno, puesto que quienes poseen menor deseabilidad social comenzaban hablando desde una postura en contra de la vejez. Esto provocaba que quienes estaban a favor o en contra, se integraban a la conversación y opinar frente al tema. Al ser un grupo diverso, existieron posiciones en cuanto a prejuicios positivos y negativos sobre la vejez, en donde los más representativos fueron la existencia de enfermedades invalidantes, abandono, inseguridad frente al bienestar en la propia vejez, versus lo hogareños, afables y cercanos a los nietos que pueden llegar a ser en una misma concepción de adulto mayor.

\section{Conclusiones}

Se puede concluir que existen representaciones sociales negativas de la vejez, a través de sus dimensiones explícitas e implícitas en el discurso de estudiantes universitarios de la Universidad Católica Silva Henríquez. Éstas pueden variar su nivel de consciencia en el sujeto, sin embargo, es un hecho que la representación social de la vejez predomina de manera negativa en el contexto social donde se insertan los participantes. El grado de ser consciente frente al propio discurso se dio de manera diferente entre las personas; es decir, existió una minoría que directamente afirmaba sentirse o ser prejuicioso, como también quienes hacían salvedades o especificaba con casos excepcionales de adultos mayores que escapaban a la norma generalizada por ellos.

De igual modo, existieron características deseables en cuanto a ser adulto mayor, donde predominó la experiencia y ser responsable, sin embargo, se dieron a conocer en menor medida 
en comparación con los aspectos poco deseables o negativos. Con ello, se da cuenta de que la representación social negativa de los adultos mayores está inserta en el contexto social de los participantes de manera consciente en algunas ocasiones y no consciente la mayoría del tiempo, las cuales son visibles a través de las contradicciones, ambivalencias, silencios y dibujos. Asimismo, se puede afirmar que los adultos mayores son vistos como seres incapacitados y dependientes entre otras características, demostrando con esto que los jóvenes de manera implícita no pueden evitar describir al adulto mayor con características puramente negativas.

Además, durante esta investigación se realizó una innovación metodológica donde se adaptó el Test de la figura humana de Machover, en el cual se solicitó representar la figura de un adulto mayor. Este método contribuyó especialmente en el análisis del prejuicio a nivel implícito, por lo que a partir de aquí sería interesante formalizar la validación de esta técnica experimental en cuanto al análisis implícito de prejuicios para diferentes grupos socialmente discriminados. Además, cabe resaltar que durante esta instancia las barreras de la deseabilidad social y el manejo de la impresión bajaron considerablemente, exponiendo casi con total libertad el verdadero pensamiento acerca de los adultos mayores y conseguir con gran precisión contrastarla con la figura humana.

Estas representaciones sociales no sólo actuarían en contra de los propios adultos mayores provocándose un estigma asociado a la profecía auto cumplida, sino que al parecer podrían generar en las futuras generaciones malestar y temor por llegar a esa etapa, teniendo a la base la percepción de elementos perturbadores para los jóvenes como el deterioro físico, mental y la necesidad de soportes, entre otros. El hecho de presentar al adulto mayor como una proyección propia a futuro, provoca temor y ansiedad, lo que daría paso a la evitación de hablar de dicho tema. Por otra parte, cabe destacar que las personas que convivieron en algún momento de sus 
vidas con familiares adultos mayores, presentaron una disminución en la presencia de prejuicios en el discurso.

Finalmente, este estudio pretende invitar al lector a la reflexión acerca de si los jóvenes estudiantes interiorizan de manera congruente a nivel explícito e implícito la representación social de la vejez, puesto que, en un aparente discurso positivo hacia la vejez, podría dar paso a prejuicios encubiertos los cuales probablemente no sean emitidos de forma consciente y es probable que a nivel cognitivo sean parte de sus representaciones sociales de la vejez. Además, fomentar el interés por educarse frente al área de la psicogerontología y ejercer en ella lejos de los mitos y construcciones sociales negativas, ya la mayoría de los entrevistados no logro visibilizar un adulto mayor distinto a la figura de abuelo o abuela deteriorado, enfermo y representado como una carga para el núcleo familiar sobre todo a nivel proyectivo en los dibujos de figuras humanas.

\section{Referencias}

Acosta, T. \& Domínguez A., (2014). Acta de Investigación Psicológica - Psychological Research Records, Vol. 4, $\quad \mathrm{N}^{\circ} \quad 2, \quad$ pp. 1535-1553. Recuperado de: http://www.redalyc.org/pdf/3589/358933347007.pdf

Angosto, J. \& Martínez, C. (2004). Dimensiones y determinantes de la intención de contacto hacia exogrupos, Vol. 38, $\mathrm{N}^{\circ}$ 2, pp. 181-190. Recuperado de http://www.academia.edu/18710282/Dimensiones_y_determinantes_de_la_intenci\%C3 \%B3n_de_contacto_hacia_exogrupos.

Araya. M. \& Villena. F, (2006). Hacia una pedagogía del encuentro cultural: discriminación y racismo, Serie Cuadernos para la ciudadanía, No 6, Costa Rica; Editorial Universidad de Costa Rica. 
Colegio de psicólogos de Chile. (1999). Código de Ética Profesional. Recuperado de http://colegiopsicologos.cl/web_cpc/wp-content/uploads/2014/10/CODIGO-DE-ETICAPROFESIONAL-VIGENTE.pdf

Cuñat, R. (2007). Aplicación de la Teoría Fundamentada (Grounded Theory) al estudio del proceso de creación de empresas, Revista Decisiones Globales, pp. 1-13. Recuperado de: http://www.ugr.es/ erivera/PaginaDocencia/Posgrado/Documentos/AlpicacionTeoriaFun damentada.pdf

Domínguez, A., Aguilera, S., Acosta, T., Navarro, G. y Ruiz, Z. (2012). La Deseabilidad Social Revalorada: Más que una Distorsión, una Necesidad de Aprobación social, Revista Acta de Investigación Psicológica, Vol. 2, $\mathrm{N}^{\circ}$ 3, pp. 808-824. Recuperado de: http://www.psicologia.unam.mx/documentos/pdf/actas_ip/2012/articulos_c/AIP_Faculta d_de_Psicologia_UNAM_23_808_824_La_Deseabilidad_Social_Revalorada_mas_que_ distorcion_una_necesidad.pdf

Flick, U. (2007). Introducción a la Investigación Cualitativa. 2da edición, Morata; Madrid.

Hernández, R., Fernández, C. \& Baptista, P. (2006). Metodología de la Investigación, 4ta ed., México; McGraw Hill.

Jorquera, P. (2010). Vejez y envejecimiento: Imaginarios sociales presentes en los textos escolares oficiales del Ministerio de educación chileno. Revista Mad. Revista del Magíster en Análisis Sistémico Aplicado a la Sociedad, № 22, pp. 132-165.

Losada Baltar, A. (2004). Edadismo: consecuencias de los estereotipos, del prejuicio y la discriminación en la atención a las personas mayores. Algunas pautas para la intervención. 
Montes-Berges, B., \& Moya, M. (2006). Medidas implícitas y explícitas de discriminación y prejuicio, Universidad de Jaén, Servicio de Publicaciones e Intercambio; Universidad de Jaén.

Osorio, P. (2008). Envejecer en el siglo XXI en América Latina. En: Granda, J. (Ed.) Pobreza, exclusión y desigualdad. Quito; FLACSO Andes.

Portuondo, J. (2007). La Figura humana. Test Proyectivo de Karen Machover, Biblioteca Nueva; Madrid.

Rodríguez, G., Gil, J., \& García, E., (1996). Metodología de la Investigación Cualitativa, Aljibe; Málaga.

SENAMA. (2013). Inclusión y exclusión social de las personas mayores en Chile. Tercera Encuesta Nacional de Inclusión y Exclusión social, Santiago; Servicio Nacional del Adulto Mayor.

Thumala, D., Arnold, M., Massad, C. \& Herrera, F. (2015). Inclusión y Exclusión social de las personas mayores en Chile. Recuperado de http://www.senama.gob.cl/storage/docs/Cuarta-Encuesta-Nacional-Inclusion-ExclusionSocial-de-las-Personas-Mayores-en-Chile-2015.pdf

Thumala, D. (2011). Formas de afrontamiento a las pérdidas asociadas al envejecer, (Tesis doctoral, Universidad de Chile, Santiago). Recuperado de http://repositorio.uchile.cl/tesis/uchile/2011/cs-thumala_d/pdfAmont/cs-thumala_d.pdf

Ungaretti, J. Etchezahar, E. \& Simkin, H (2012). El estudio del prejuicio desde una perspectiva psicológica: cuatro periodos histórico- conceptuales para la comprensión del fenómeno. Revista Calidad de Vida Universidad de Flores, $\mathrm{N}^{\circ} 8$, pp. 13-30. 
Zavala, M., Vidal, D., Castro, M., Quiroga, P. \& Klassen, G. (2006). Funcionamiento Social de los adultos mayores, Revista Ciencia y Enfermería, Vol. 12, № 2, pp. 53-62.

Recepción: 2 de septiembre de 2019

Última Revisión: 22 de diciembre de 2019

Aceptación Final: 30 de diciembre de 2019 